CVIA

REVIEW ARTICLE

pISSN 2508-707X / elSSN 2508-7088 https://doi.org/10.22468/cvia.2018.00199 CVIA 2018;2(4):166-175

Received: September 19, 2018

Revised: October 2, 2018

Accepted: October 10, 2018

Corresponding author

Dong Hyun Yang, MD, PhD

Department of Radiology and

Research Institute of Radiology,

Cardiac Imaging Center,

Asan Medical Center, University of Ulsan

College of Medicine,

88 Olympic-ro 43-gil, Songpa-gu,

Seoul 05505, Korea

Tel: 86-2-3010-5820

Fax: 86-2-476-8127

E-mail: donghyun.yang@gmail.com

\section{Applications of Three-Dimensional Printing in Cardiovascular Surgery: A Case-Based Review}

\author{
Dong Hyun Yang ${ }^{1,2}$, Sang-Hyub Park ${ }^{1}$, Koeun Lee², Taehun Kim², \\ Joon Bum Kim³, Tae-Jin Yun³, Guk Bae Kim, Namkug Kim², \\ 'Department of Radiology and Research Institute of Radiology, Cardiac Imaging Center, \\ Asan Medical Center, University of Ulsan College of Medicine, Seoul, Korea \\ ${ }^{2}$ Medical Imaging and Intelligent Reality Laboratory, Asan Medical Center, \\ University of Ulsan College of Medicine, Seoul, Korea \\ ${ }^{3}$ Departments of Thoracic and Cardiovascular Surgery, ${ }^{5}$ Convergence Medicine, \\ Asan Medical Center, University of Ulsan College of Medicine, Seoul, Korea \\ ${ }^{4}$ Anymedi Inc., Seoul, Korea
}

\begin{abstract}
Modern techniques of image acquisition, post-processing, computer-aided design, and three-dimensional (3D) printing allow for more precise understanding of the complex anatomy in various cardiovascular diseases. The 3D printing for cardiovascular disease may be used as an appropriate educational tool for teaching complex surgical techniques through handson simulation of surgery. Moreover, a patient-specific surgical graft could be constructed in accordance with the 3D printed model before the surgery. In this review, we introduce examples of clinical applications of various types of 3D printing based on specific cases and clinical application scenarios of 3D printing. We also discuss the limitations and clinically unmet needs of 3D printing for the preoperative planning of patients with cardiovascular disease.
\end{abstract}

Key words 3D printing $\cdot$ Heart defect, congenital $\cdot$ Aorta Cardiac imaging techniques.

\section{INTRODUCTION}

The development of cardiac imaging techniques has increased the importance of preoperative information obtained from CT or MRI when planning cardiovascular surgery [1-8]. Cardiac images intuitively demonstrate the morphological changes of critical structures to a cardiovascular surgeon, and thus help the surgeon to plan the surgery in detail and to prevent unexpected complications [5,6]. In recent years, three-dimensional (3D) printing has been introduced to provide more intuitive anatomical information than images, and this makes it possible to provide not only visual information but also tactile and spatial information $[9,10]$. It is also possible to educate many surgeons regarding complicated surgical procedures that can be used in rare cases. To date, many authors have reported the application of 3D printing to a variety of clinical cases, including patients with congenital heart disease (CHD), vascular disease, valve dis-

(c) This is an Open Access article distributed under the terms of the Creative Commons Attribution Non-Commercial License (https://creativecommons.org/licenses/by$\mathrm{nc} / 4.0$ ) which permits unrestricted non-commercial use, distribution, and reproduction in any medium, provided the original work is properly cited. ease, cardiac tumors, guidance for cardiac ablation therapy, and myocardial disease [9-12]. There are also many differences in the materials and output methods applied for the purpose of 3D printing [10]. Asan Medical Center in Seoul, Korea has been applying 3D printing for preoperative planning in the various cardiovascular diseases since 2015. As seen in previously published manuscripts [11,12], 3D printing methods and materials are applied differently depending on the type of disease and the purpose of use. In this review, we introduce clinical application examples of various 3D printing approaches based on specific cases and clinical applications of 3D printing. We would also like to discuss the limitations and clinical unmet needs regarding 3D printing for the preoperative planning of cardiovascular disease.

\section{WORKFLOW OF 3D PRINTING}

The process of 3D printing may be divided into three steps as follows, although there are some differences in the usage terms as compared to previously published manuscripts: 1) image acquisition and post-processing; 2) computer-aided design; 
and 3) printing and preparation for clinical use [10-13].

\section{Image acquisition and post-processing}

As 3D printing is made using 'images', it is very important to obtain the original image with high quality, and cardiovascular imaging experts must confirm the accuracy of the image [13]. MRI or echocardiography may be used, but in many published reports, contrast-enhanced CT has been used as a source image for 3D printing [12]. In general, iso-cubic voxel spacing for 3D image post-processing is preferred when obtaining CT images. Sometimes obtaining images with homogeneous contrast enhancement may be important for the segmentation of images. For example, when $3 \mathrm{D}$ printing of the right ventricle and the right atrium is required, it is important to keep the contrast enhancement of the right-sided chambers uniform for efficient image segmentation. For this purpose, it may be helpful to use bi-phasic or tri-phasic contrast enhancement techniques [11]. In patients with heart disease, it is often necessary to perform an electrocardiography (ECG)-gated scan in order to prevent artifacts caused by cardiac movements. In addition, depending on the target structure, the proper cardiac phase, i.e., systole or diastole, should be chosen. For example, in the case of hypertrophic cardiomyopathy, it is preferable to obtain diastolic images, because hypertrophied myocardium and papillary muscle may not be well distinguished in systolic images [9]. Conversely, if you wish to evaluate the appearance of a valve opening in aortic stenosis, systolic imaging may be better.

Image post-processing comprises the segmentation and surface modeling of the target organ or structure [11-13]. Thresholding, seeded region-growing, graph cuts, and volume sculpting can be used to segment the desired part of the heart and vessels. In $3 \mathrm{D}$ printing, coloring important structures with different colors can be better than using a single color for understanding the abnormalities in complex cardiac anomalies. For this purpose, it is necessary to separate each structure individually during image segmentation (Fig. 1). In order to use postprocessed images for $3 \mathrm{D}$ printing, it is necessary to convert them into a file format capable of 3D mesh generation, such as a STereoLithography or Standard Tessellation Language (STL), Virtual Reality Modeling Language (VRML) or Object (OBJ) file [11]. Therefore, it is necessary to confirm whether the workstation can export the divided image area as a file capable of 3D mesh generation. Some open source programs, such as Slicer (www.slicer.org) and ITK-SNAP (http://www.itksnap.org), provide various tools for image post-processing. At our institution, the post-processing is performed using various types of commercial software or in-house software (A-View Cardiac, Asan Medical Center, Seoul, Korea), depending on the purpose of the examination. Image post-processing can be performed by an experienced researcher or radiology technologist who can manage time-consuming tasks, although a cardiovascular imaging expert must confirm the end result in order to ensure the accuracy of the data.

\section{Computer-aided design}

After obtaining the file, i.e. an STL file, for 3D mesh modeling, it is necessary to modify the structure to fit the purpose of $3 \mathrm{D}$ printing or to add new artificial markers. For example, adding an artificial ring demonstrating a ventricular septal defect (VSD) or valve annulus in complex CHD can help the surgeon to understand the interrelationships between the anatomical structures. Another example of computer-aided design is to
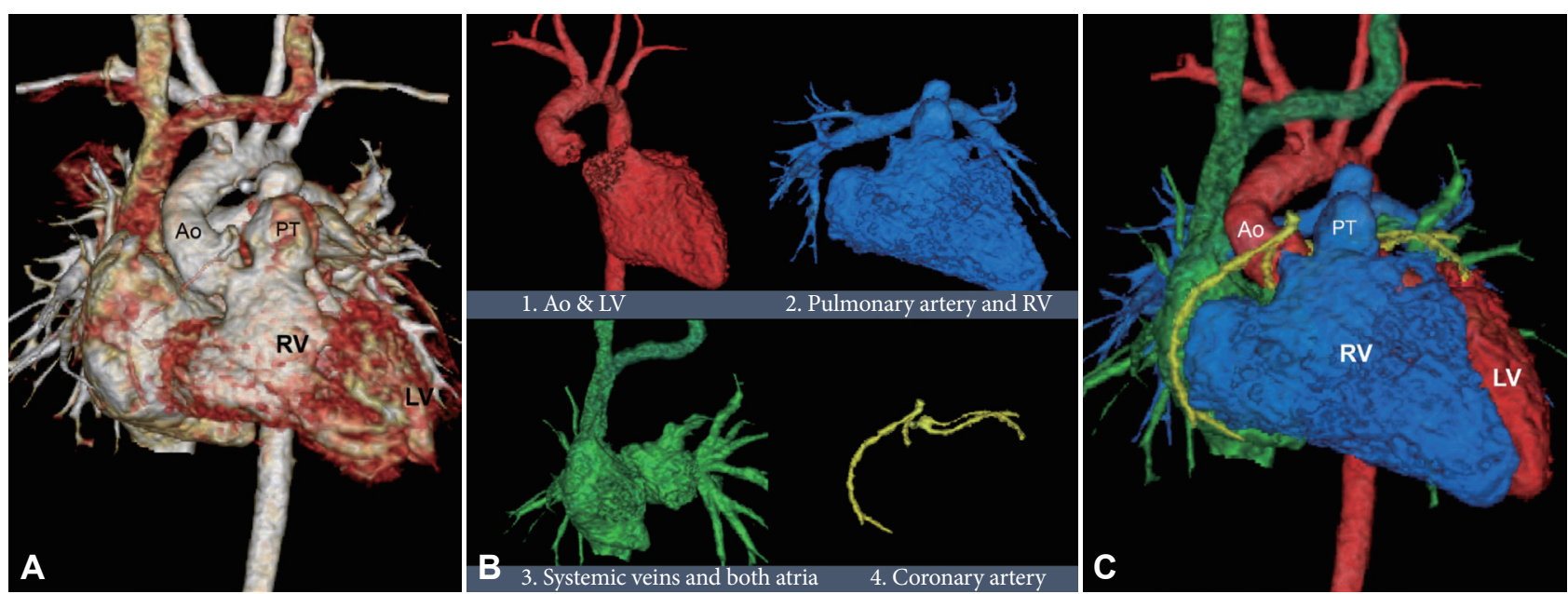

Fig. 1. Image segmentation process from a four-month-old girl. (A) Typical volume rendering image in a four-month-old girl with a double outlet right ventricle and doubly committed perimembranous ventricular septal defect (not shown). (B) Image segmentation of each anatomical structure in a commercially available software program (Advantage Windows 4.6; GE Healthcare, Milwaukee, WI, USA). In this software, a Dicom file can be exported to STL, and this is performed separately for each anatomical structure. (C) Fusion of each anatomical structure in a volume rendering image. Ao: aorta, PT: pulmonary trunk, RV: right ventricle, LV: left ventricle, STL: STereoLithography or Standard Tessellation Language. 
create a virtual ventricular wall when we wish to express the endoluminal view of the ventricle. After splitting the enhanced ventricular chamber, applying pixel dilation approximately $2 \mathrm{~mm}$ outward, and subtracting the original chamber, an endoluminal view with a virtual myocardium can be expressed (Fig. 2). This method is often used because it saves time and effort in image segmentation compared to the direct segmentation of the myocardium itself. Once the anatomical structures have been modified or artifacts have been added, smoothing of an object must be performed before printing. In addition, a surface defect created during mesh generation must also be corrected. If necessary, applying different colors between structures can help to increase the understanding of the operator.

\section{Printing and preparation for clinical use}

The first step of printing an object would be the determination of an appropriate printing material and a printer suitable for its clinical use [10]. Since the budget for 3D printing depends on the material and printer, it is important to identify the purpose of its use and to decide on the appropriate materials. For example, if the purpose is to identify only the interrelationships of the anatomical structures, using relatively inexpensive materials is more cost-effective. On the other hand, if the surgeon wishes to perform a simulated operation on the $3 \mathrm{D}$ printing, it is important to ensure that the $3 \mathrm{D}$ printing materials are not eas- ily torn, even if surgical suturing is performed on it. These rubber-like and tough printing materials and their printers are usually expensive [10]. In the case of complex CHD, 3D printing typically takes 3-10 hours to build a heart model, depending on its size [11]. After the 3D printing is completely built, the supporting material should be removed for clinical use. Removal of the supporting material could be performed by hand, by washing with high-pressure water, or by dissolution in a solvent. The overall process of printing and preparation of $3 \mathrm{D}$ printing for clinical use is demonstrated in Supplementary Movie 1 (in the online-only Data Supplement).

\section{CASE DEMONSTRATION}

\section{Complex congenital heart disease}

Using 3D printing for complex CHD is one of the most common applications $[11,12,14]$. The use of $3 \mathrm{D}$ printing in complex $\mathrm{CHD}$ can reduce the degree of the surgeon's uncertainty in the preoperative anatomical evaluation and, if necessary, allow handson surgery to be used for the actual surgery $[11,14]$. ECG-gated CT or MR angiography are the most commonly used imaging methods for the $3 \mathrm{D}$ printing. For a better understanding of the anatomy, graphically designed valvular annuli could be added during the computer modeling process, as suggested by Yoo et al. $[11,14]$. Two types of 3D printing have been widely applied
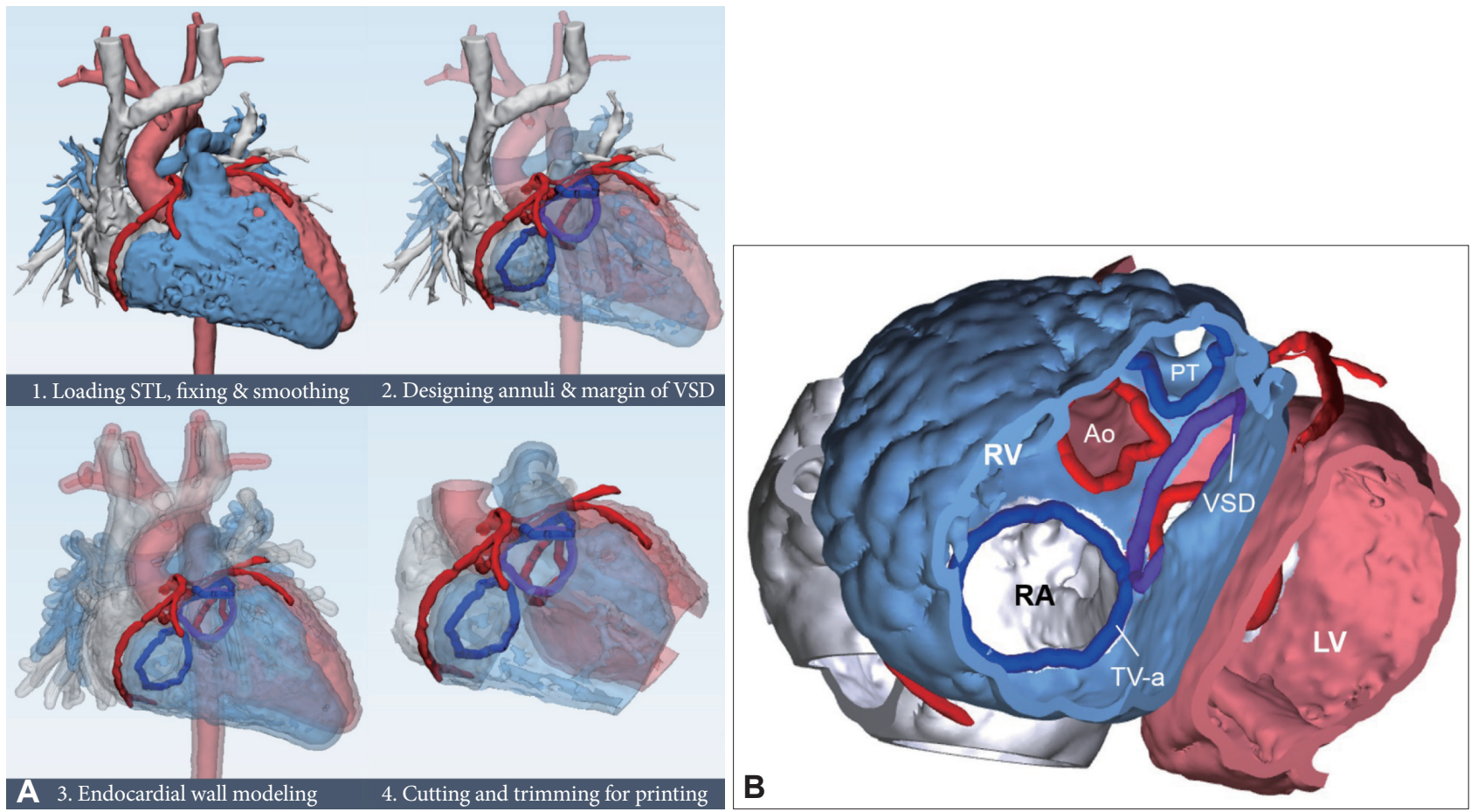

Fig. 2. Computer-aided design for the patient shown in Fig. 1. (A) The typical process of computer-aided design in commercially available software (Materialise 3-matic, Materialise, Leuven, Belgium). (B) Inferior view of the three-dimensional model shows a spatial relationship between the VSD and the valvular annuli. STL: STereoLithography or Standard Tessellation Language, VSD: ventricular septal defect, Ao: aorta, PT: pulmonary trunk, RV: right ventricle, LV: left ventricle, RA: right atrium, TV-a: tricuspid valvular annulus. 

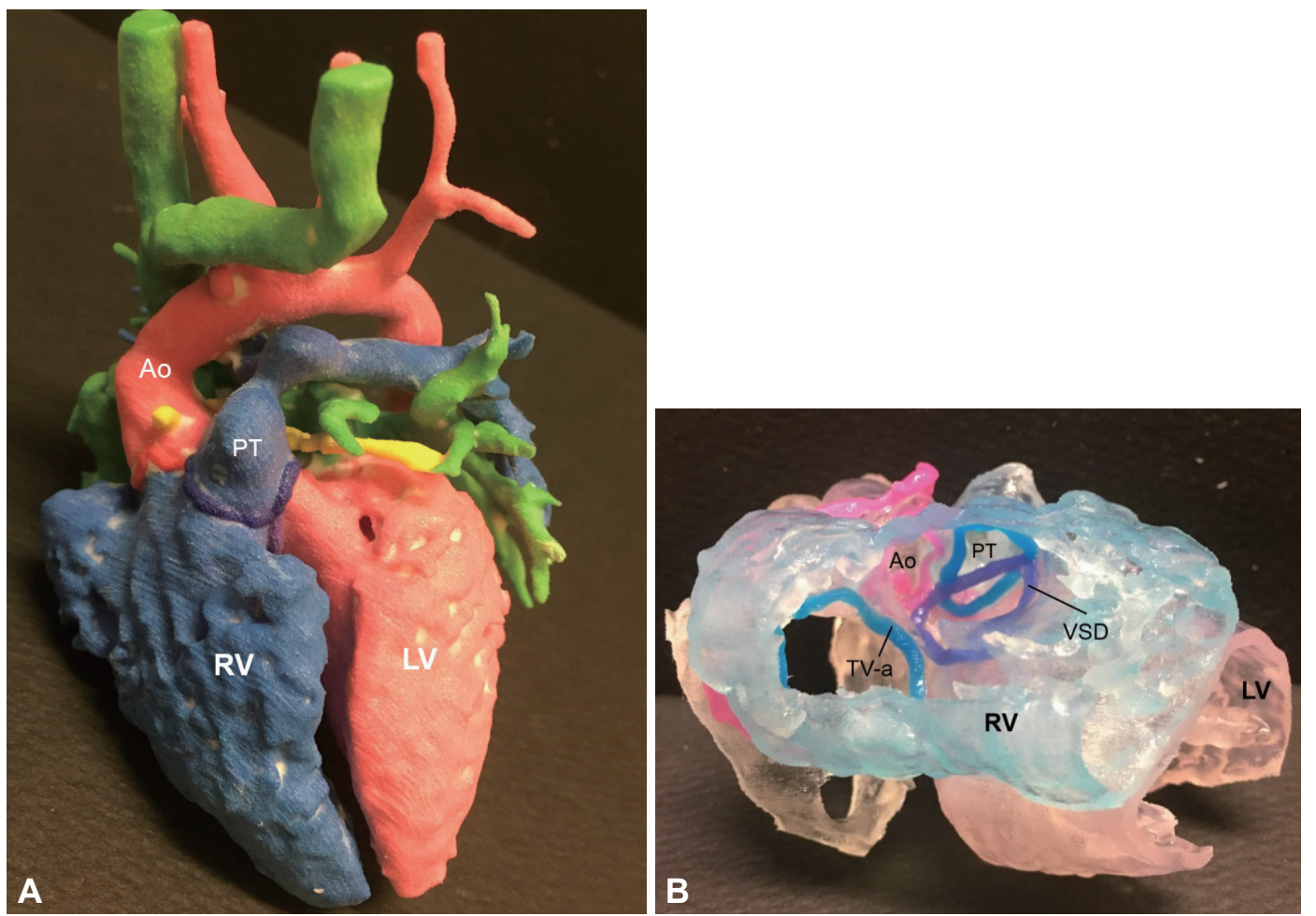

Fig. 3. 3D printings from the patient shown in Fig. 1. (A) Cast model of the blood pool and (B) endocardial wall model of 3D printings clearly show the intra- and extra-cardiac anatomy in the patient with a double outlet right ventricle and doubly committed VSD. 3D: three-dimensional, Ao: aorta, PT: pulmonary trunk, RV: right ventricle, LV: left ventricle, TV-a: tricuspid valvular annulus, VSD: ventricular septal defect.

for complex CHD [11]: cast models of the blood pool (Fig. 3A) and endocardial wall models (Fig. 3B) representing the endocardial surface. A cast model is useful for evaluating the overall anatomical structure of the heart and neighboring great vessels. A rigid plastic or gypsum-like material, which is relatively inexpensive, may be sufficient for a cast model. An endocardial wall model allows the detailed evaluation of intracardiac structures. With an endocardial model, it is easy to understand the relationship between structures within the heart, such as a VSD, papillary muscle, and valvular annuli. For an endocardial model, a soft and rubber-like material may be suitable for a better tactile sense and the need for surgical practice. In an endocardial wall model, an appropriate cutting plane on the atrial and ventricular sides should be designed before the printing in order to provide an in-depth view of the intracardiac anatomy. We usually provide one cast model (Projet 460 printer and Visijet PXL Core powder, Visijet PXL clear binder and color bonds; 3D Systems, Rock Hill, SC, USA) and two endocardial models (Objet Connex Series printer and TangoPlus FullCure resin, Stratasys Ltd., Eden Prairie, MN, USA) for a patient with complex CHD.

Table 1 shows the indications for 3D printing performed at our institution for preoperative planning of CHD since 2017. The most common indication was to confirm the feasibility of intraventricular baffling in the double outlet right ventricle (DORV; $24 / 35$ cases, $68 \%$ ). In some patients with DORV, the possibility
Table 1. Indications for three-dimensional printing applications in $\mathrm{CHD}$ at Asan Medical Center since 2017 (35 cases)

\begin{tabular}{lc}
\hline \multicolumn{1}{c}{ CHD } & $\begin{array}{c}\text { Number } \\
\text { of cases (\%) }\end{array}$ \\
\hline $\begin{array}{l}\text { Double outlet right ventricle } \\
\text { Transposition of the great arteries, complete and } \\
\text { congenitally corrected }\end{array}$ & $24(68)$ \\
Interrupted aortic arch & $1(20)$ \\
Criss-cross heart or superoinferior ventricles & $1(3)^{*}$ \\
Aortic atresia & $1(3)$ \\
Left ventricular apical aneurysm & $1(3)$ \\
\hline
\end{tabular}

*This case also had a double outlet right ventricle. CHD: congenital heart disease

of intraventricular baffling was difficult to determine even if 3D CT images and echocardiography were reviewed. In such a case, communication between imaging specialists and a surgeon was often difficult because of the ambiguous association between the VSD, valvular annuli, and the outlet septum. However, after applying $3 \mathrm{D}$ printing, particularly the endocardial wall model, the surgeon was better able to determine the feasibility of intraventricular baffling after seeing and directly manipulating the $3 \mathrm{D}$ printing (Supplementary Movie 2 in the online-only Data Supplement). This has resulted in a dramatic decrease in timeconsuming arguments and complicated anatomical descriptions that might have occurred in the heart team conference. 
Another important indication of $3 \mathrm{D}$ printing, although it is very rare, is a criss-cross heart; other commonly used terms are 'superior-inferior ventricles' or 'twisted atrioventricular connection' $[15,16]$. This peculiar heart often confuses an inexperienced radiologist. The relationship between the chambers and the great vessels is complex and varies from one patient to another depending on the degree and direction of the so-called 'twisted' ventricles. Therefore, it may be very difficult to understand absolutely the anatomical information when using the conventional imaging methods, and even if a radiologist understands it, it is difficult to explain it to their colleagues. As 3D printing is intuitive and does not require so-called 'mental reconstruction', it can be of great help for making an accurate diagnosis and for communication of the medical staff (Fig. 4, Supplementary Movie 3 in the online-only Data Supplement).

Finally, an important use of 3D printing in $\mathrm{CHD}$ is surgical practice. In particular, it is useful when the surgical technique is complicated. Yoo et al. [11] introduced an application of 3D printing for the training of inexperienced surgeons to teach them how to perform the Norwood surgery in hypoplastic left heart syndrome in Yoo's outstanding review article. In our institute, our heart team held a workshop to teach the aortic translocation (Nikaidoh) procedure for patients with d-transposition of the great arteries (d-TGA), VSD, and pulmonary stenosis. The patients' outcomes were then far superior to those of the conventional Rastelli procedure in this patient group with d-TGA, VSD, and pulmonary stenosis [17]. Nonetheless, the overall experience with this Nikaidoh surgery is somewhat limited due to its technical complexity and the relatively sparse patient group who are suitable for this surgery [17]. We created a surgical model in a two-year-old's boy with d-TGA, VSD, and subpulmonary narrowing (Fig. 5). To create a model capable of hands-on surgery, a printed rubber-like material needed to be improved so that it would not substantially tear with repeated surgical sutures. To do this, we coated the surface of the printed model with appropriate chemicals. Eventually, many surgeons in the workshop were able to successfully and directly practice the Nikaidoh procedure on the model (Supplementary Movie 4 in the online-only Data Supplement).

\section{Thoracoabdominal aorta repair surgery}

Another common indication for cardiovascular 3D printing at our institution is aortic disease. The main purpose is to make a patient-specific, branched surgical graft during thoracoabdominal aorta (TAA) repair surgery. TAA repair is a very challenging operation in which the mortality and disabling complications are reported to be $7.5 \%$ and $10.0 \%$, respectively, even when the operation is performed by an expert group [18]. One cause of such a high surgical risk is that vital branch arteries must be transferred to a new surgical graft, which is time-consuming and complicated [19]. To overcome this, we applied 3D printing to design a patient-specific, branched, surgical aortic graft. Based on a 3D CT image, a 'virtual graft' of the aorta and its main branches was generated using computer modeling. The important branches included the celiac trunk, superior mesenteric artery, renal arteries, and prominent intercostal arteries at T8 or lower levels. The virtual graft was made at the actual size using $3 \mathrm{D}$ printing and was sterilized for use in the operating room. Then, the patient-specific branched graft was constructed in accordance with the 3D-printed virtual graft before skin incision, using a commercially available surgical graft (Gelweave Coselli thoracoabdominal graft; Vascuteck Ltd., Renfrewshire, UK) (Sup-
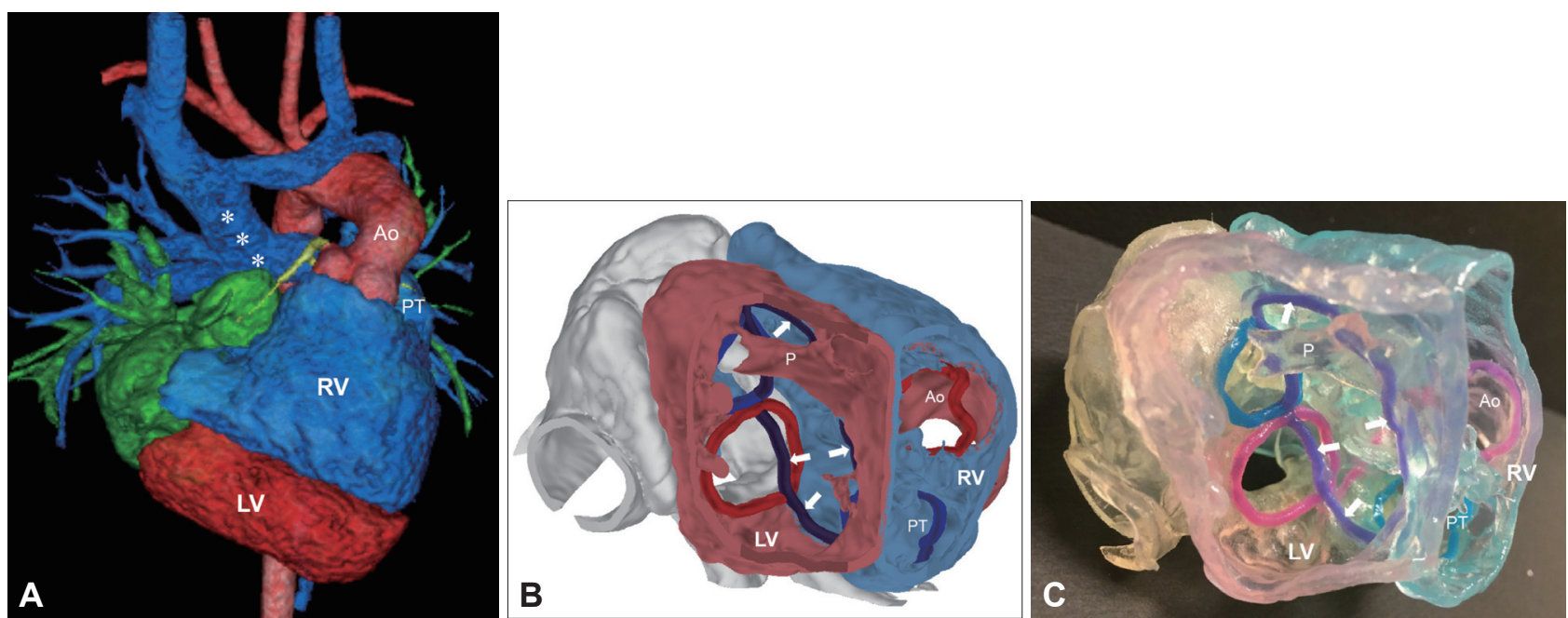

Fig. 4. Twisted (clockwise) DORV with a huge VSD in two-year-old boy. (A) Multi-colored volume rendering image, (B) cardiac chamber views of computer-aided design, and $(\mathrm{C})$ an endocardial wall model of three-dimensional printing show the very complex anatomy of cardiac chambers and great vessels. Note the pulsatile, bidirectional cavopulmonary shunts (asterisks), D-malposition of great arteries, twisted (clockwise) DORV with huge VSD (arrows), and overriding of the tricuspid valve and mitral valve. DORV: double outlet right ventricle, VSD: ventricular septal defect, Ao: aorta, PT: pulmonary trunk, RV: right ventricle, LV: left ventricle, P: papillary muscle. 

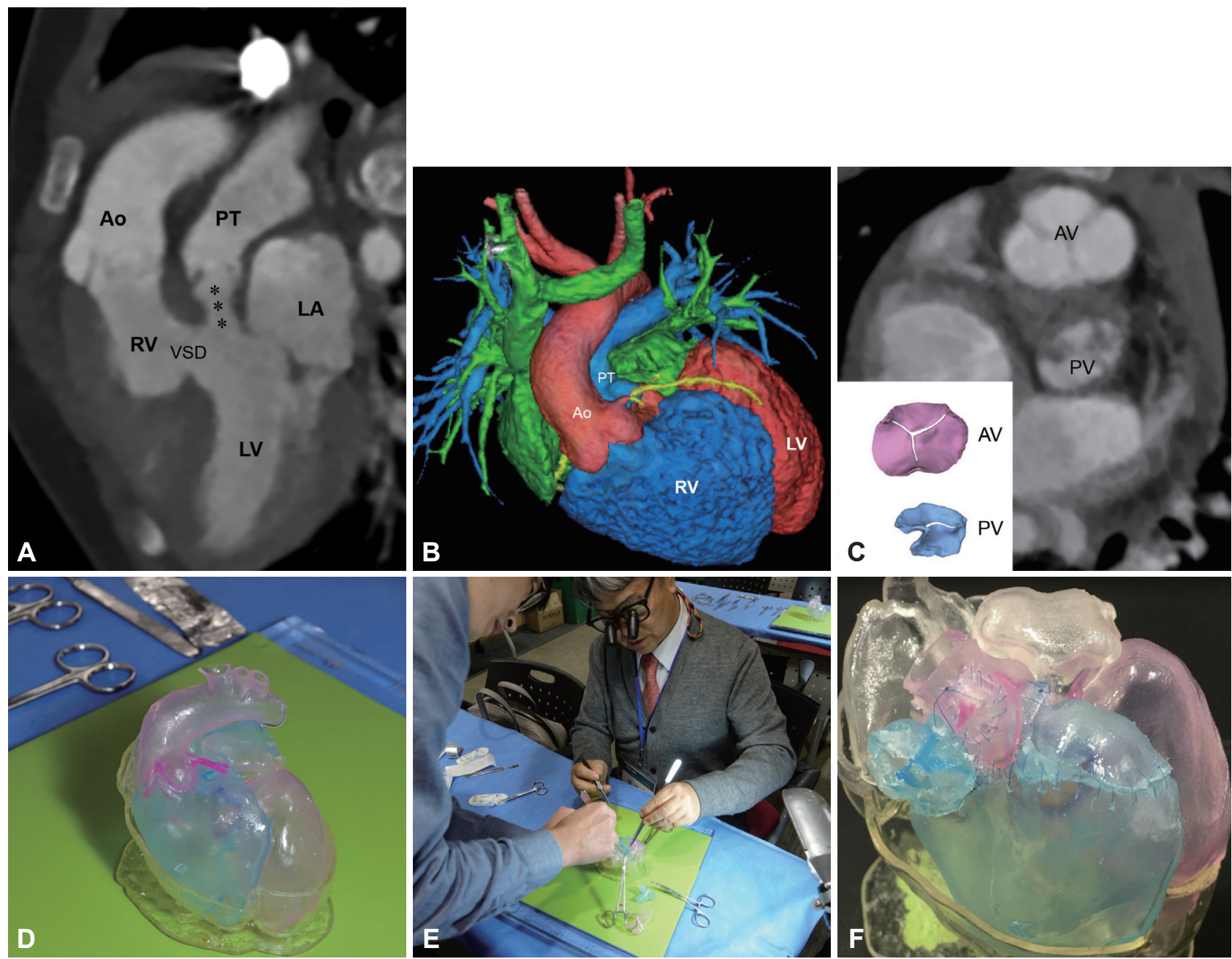

Fig. 5. 3D printing for hands-on surgery in a three-year-old boy with d-TGA, VSD, and subpulmonary narrowing. (A) Oblique coronal and (B) volume rendering images show d-TGA, VSD, and subpulmonary narrowing (asterisks). (C) For more realistic printing, cardiac valves were designed based on a CT image. Note the bicuspid pulmonary valve. ( $D$ and $E$ ) 3D printing for hands-on surgery was used for an educational symposium to teach the aortic translocation (Nikaidoh) procedure. (F) An aortic translocation procedure was successfully performed on the 3D printing. d-TGA: d-transposition of the great arteries, VSD: ventricular septal defect, 3D: three-dimensional, Ao: aorta, PT: pulmonary trunk, RV: right ventricle, LV: left ventricle, LA: left atrium, AV: aortic valve, PV: pulmonary valve.

plementary Movie 5 in the online-only Data Supplement). At this time, a virtual graft made by $3 \mathrm{D}$ printing was used to determine the position and direction of the branches. Since 2017, eight cases of TAA repair were performed under the $3 \mathrm{D}$ printing guide and all of them could be done safely without postoperative morbidity or motility. In particular, 3D printing has been successfully used in patients with severe scoliosis with aorta disease (Fig. 6).

\section{Aortic valve-sparing operation}

Although aortic valve (AV)-sparing root replacement surgery has been developed to manage aortic root dilatation with significant aortic regurgitation [20], there is still much controversy regarding standardizing the surgical method for the root reconstruction [21,22]. Among the many difficulties of this procedure, 'asymmetricity of the root' could be a major obstacle for reconstructing a competent $\mathrm{AV}$ function [23,24]. For a more tailored and individualized surgical approach, 3D printing might be applied for preoperative planning of the AV-sparing root reconstruction surgery (Fig. 7).

\section{Hypertrophic cardiomyopathy}

Surgical myectomy, which is required in hypertrophic cardiomyopathy patients with severe left ventricular (LV) outflow tract obstruction, is a conceptually simple procedure. Nonetheless, a complex LV outflow tract anatomy, combined anomalies of the papillary muscle, and limited surgical field of view through the aortic root and cardiac apex may increase the risk of surgical morbidity or incomplete myocardial resection. A 3D printed myocardial model may provide more intuitive information on the LV geometry and allow for precise preoperative simulation of the surgical myectomy (Fig. 8) [9,25]. 

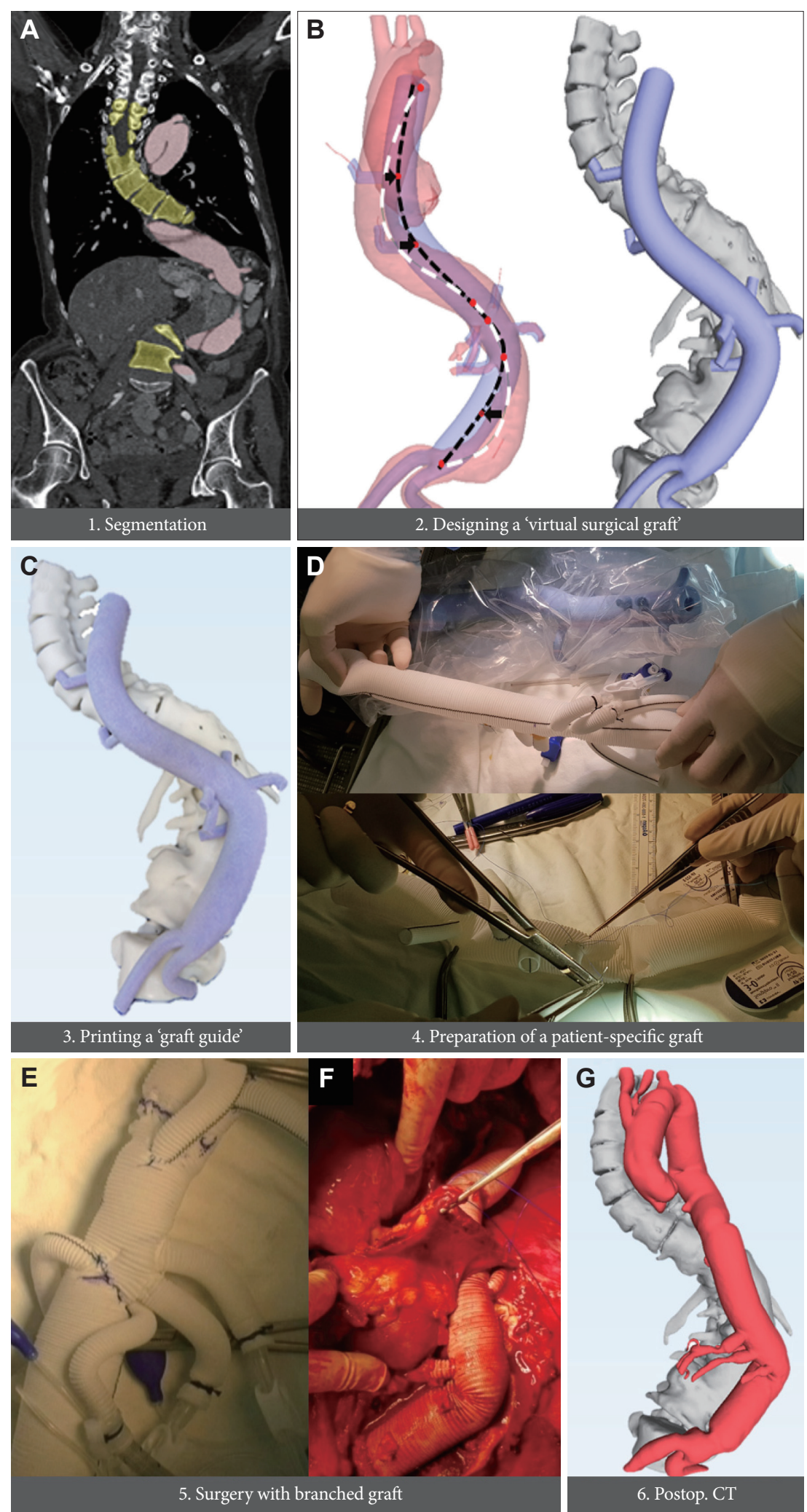

Fig. 6. 3D printing for construction of a patient-specific surgical graft in a 58-year-old woman with Marfan syndrome. (A) A preoperative coronal CT image shows type B aortic dissection and severe scoliosis. (B and C) Based on the patient's CT data, a 'virtual surgical graft' was designed to build by $3 \mathrm{D}$ printing. A centerline of the virtual graft (arrows, black dotted line) could be slightly different with the original centerline of the dilated aorta (white dotted line). (D-E) Using a printed 'graft guide' as a reference, an actual surgical graft can be constructed before incision. ( $F$ ) Thoracoabdominal aorta repair surgery was done in this patient using the patient-specific graft. (G) Postoperative CT shows patent branches from the aortic graft. 3D: three-dimensional. 

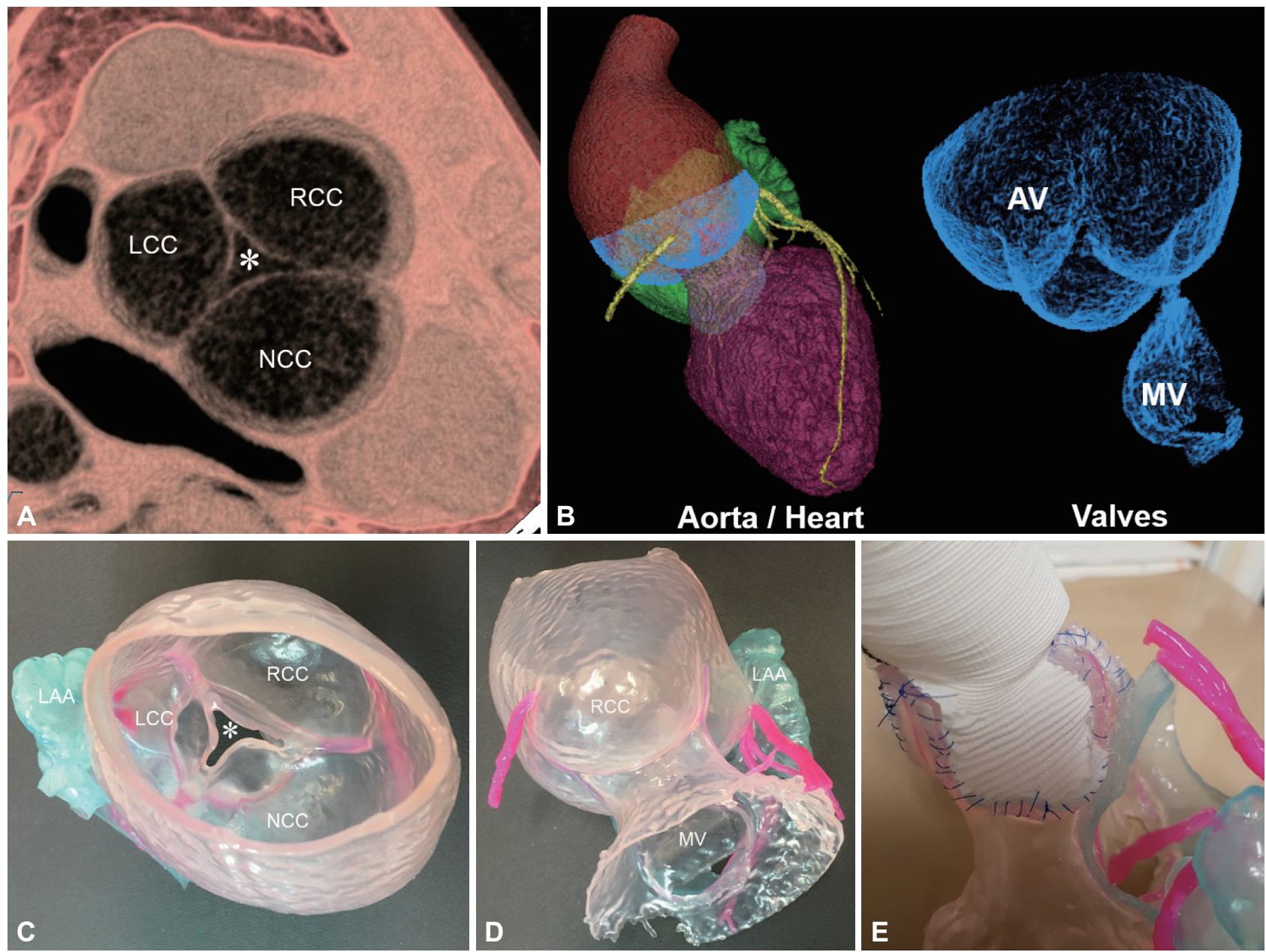

Fig. 7. 3D printing for hands-on surgery in a 29-year-old man with aortic annuloectasia, aortic regurgitation, and Marfan syndrome. (A) The surgeon's view of the volume-rendering CT image shows a large central coaptation defect (asterisk) of the AV. Note that the asymmetry in the aortic sinus (B) volume rendering images shows segmentation results of the aorta, cardiac chambers, AV, and MV. (C and D) Preoperative 3D printings show morphology of the aortic root and AV. Note the relatively thick and clumsy AV leaflet. (E) Hands-on surgery is only performed in the aortic root. Practice of the valvuloplasty could not be performed, because the 3D printing failed to represent the AV precisely. 3D: three-dimensional, RCC: right coronary sinus, NCC: non-coronary sinus, LCC: left coronary sinus, AV: aortic valve, MV: mitral valve, LAA: left atrial appendage.

\section{LIMITATIONS AND UNMET NEEDS}

With current 3D printing technology, it may be impossible to reproduce the fine cardiovascular structures, such as the valve leaflet and chordae tendinea, with similar tactile sense and strength to those of the human body (Fig. 7). To achieve precise representation of such delicate structures, not only 3D printing technology and material improvements, but also image acquisition technology, segmentation, and computer-aided design modeling should be developed $[11,12,26]$. Another important limitation is that cardiovascular $3 \mathrm{D}$ printing is very expensive. Image segmentation, computer-aided design, printing, and post-printing preparation for clinical use all require time and skillful professionals who have anatomical knowledge. In addition, costly printers and materials are required for the $3 \mathrm{D}$ printing suitable for hands-on surgical practice [11]. In a case of complex CHD, the cost of producing 3D printings, i.e., one cast model and two endocardial wall models, is approximately 1000-2000 US dollars, depending on its size, and the production period is $3-4$ days.

\section{CONCLUSION}

3D printing can be used as a highly personalized treatment method in the area of cardiovascular surgery. In a cardiovascular disease showing morphological complexity, a 3D print allows a clearer understanding of the anatomy or hands-on training before surgery. However, further development of image processing and 3D printing technology is required in order to represent a more delicate cardiovascular structure. Research is also required in order to lower the cost of 3D printing for broader clinical applications. 


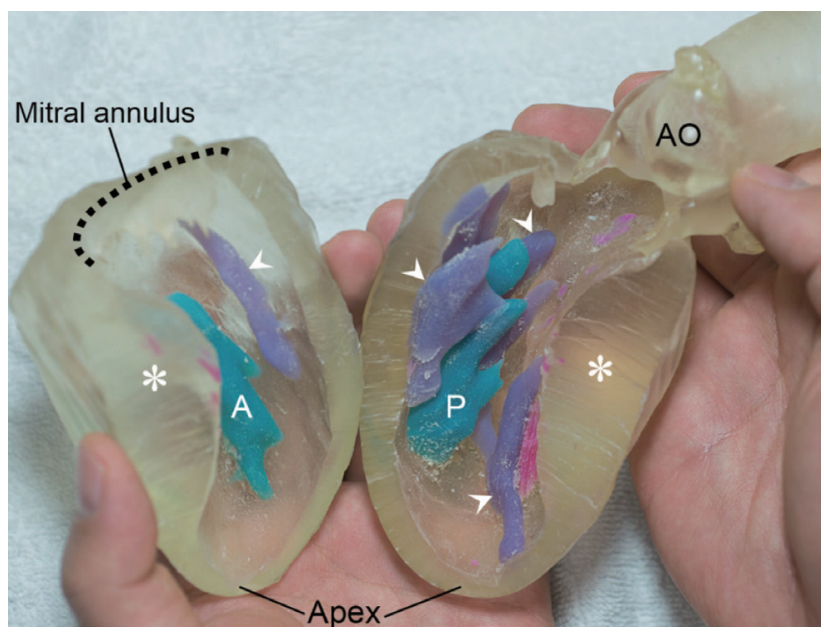

Fig. 8. Myocardial 3D printing for septal myectomy guidance in a 33-year-old woman with obstructive hypertrophy cardiomyopathy. Myocardial 3D printing shows the geometric relationship of the hypertrophied septum (asterisks), papillary muscles (A, anterior; P, posterior), and intraventricular muscle bands (arrow heads). 3D: three-dimensional, AO: aorta.

\section{Supplementary Movie Legends}

Movie 1. Process of printing and post-printing preparation for the clinical use.

Movie 2. Preoperative exploration of 3D printing. Double outlet right ventricle with doubly committed ventricular septal defect.

Movie 3. Preoperative exploration of 3D printing. Twisted double outlet right ventricle with a huge ventricular septal defect.

Movie 4. Hands-on surgery workshop using 3D printing.

Movie 5. Construction of a patient-specific branched graft using a 3D printed graft.

\section{Supplementary Materials}

The online-only Data Supplement is available with this article at https:// doi.org/10.22468/cvia.2018.00199.

\section{Conflicts of Interest}

Dong Hyun Yang and Joon Bum Kim hold stock in Anymedi Inc. (http:// anymedi.com/). Namkug Kim and Guk Bae Kim are co-founders of Anymedi Inc. (http://anymedi.com/).

\section{Acknowledgments}

This work was supported by National Research Foundation of Korea (NRF) grants funded by the Korean government (MSIP) (NRF-2016R1A1A1A05921 207) and a grant from the Korea Health Technology R\&D Project through the Korea Health Industry Development Institute (KHIDI), funded by the Ministry of Health \& Welfare, Republic of Korea (HI17C2410).

\section{REFERENCES}

1. Yang DH, Goo HW, Seo DM, Yun TJ, Park JJ, Park IS, et al. Multislice CT angiography of interrupted aortic arch. Pediatr Radiol 2008;38:89-100.

2. Koo HJ, Yang DH, Oh SY, Kang JW, Kim DH, Song JK, et al. Demonstration of mitral valve prolapse with CT for planning of mitral valve repair. Radiographics 2014;34:1537-1552.

3. Kim YJ, Yong HS, Kim SM, Kim JA, Yang DH, Hong YJ; Korean Society of Radiology; Korean Society of Cardiology. Korean guidelines for the appropriate use of cardiac CT. Korean J Radiol 2015;16:251-285.

4. Koo HJ, Kang JW, Kim JA, Kim JB, Jung SH, Choo SJ, et al. Functional classification of aortic regurgitation using cardiac computed tomography: comparison with surgical inspection. Int J Cardiovasc Imaging 2018;34: 1295-1303.

5. Koo HJ, Lee JY, Kim GH, Kang JW, Kim YH, Kim DH, et al. Paravalvular leakage in patients with prosthetic heart valves: cardiac computed tomography findings and clinical features. Eur Heart J Cardiovasc Imaging. 2018 Jan 4 [Epub]. https://doi.org/10.1093/ehjci/jex341.

6. Koo HJ, Yang DH, Kang JW, Lee JY, Kim DH, Song JM, et al. Demonstration of infective endocarditis by cardiac CT and transoesophageal echocardiography: comparison with intra-operative findings. Eur Heart J Cardiovasc Imaging 2018;19:199-207.

7. ASCI Practice Guideline Working Group, Beck KS, Kim JA, Choe YH, Hian SK, Hoe J, Hong YJ, et al. 2017 multimodality appropriate use criteria for noninvasive cardiac imaging: Expert Consensus of the Asian Society of Cardiovascular Imaging. Korean J Radiol 2017;18:871-880.

8. Song Y, Yang DH, Ó Hartaigh B, Cho SJ, Kang JW, Kim YH, et al. Geometric predictors of left ventricular outflow tract obstruction in patients with hypertrophic cardiomyopathy: a 3D computed tomography analysis. Eur Heart J Cardiovasc Imaging 2018;19:1149-1156.

9. Yang DH, Kang JW, Kim N, Song JK, Lee JW, Lim TH. Myocardial 3-dimensional printing for septal myectomy guidance in a patient with obstructive hypertrophic cardiomyopathy. Circulation 2015;132:300-301.

10. Kim GB, Lee S, Kim H, Yang DH, Kim YH, Kyung YS, et al. Three-dimensional printing: basic principles and applications in medicine and radiology. Korean J Radiol 2016;17:182-197.

11. Yoo SJ, Thabit O, Kim EK, Ide H, Yim D, Dragulescu A, et al. 3D printing in medicine of congenital heart diseases. 3D Print Med 2015;2:3.

12. Giannopoulos AA, Mitsouras D, Yoo SJ, Liu PP, Chatzizisis YS, Rybicki FJ. Applications of 3D printing in cardiovascular diseases. Nat Rev Cardiol 2016;13:701-718.

13. Mitsouras D, Liacouras P, Imanzadeh A, Giannopoulos AA, Cai T, Kumamaru KK, et al. Medical 3D printing for the radiologist. Radiographics 2015;35:1965-1988.

14. Yoo SJ, van Arsdell GS. 3D printing in surgical management of double outlet right ventricle. Front Pediatr 2018;5:289.

15. Yoo SJ, Seo JW, Lim TH, Park IS, Hong CY, Song MG, et al. Hearts with twisted atrioventricular connections: findings at MR imaging. Radiology 1993;188:109-113.

16. Seo JW, Yoo SJ, Ho SY, Lee HJ, Anderson RH. Further morphological observations on hearts with twisted atrioventricular connections (crisscross hearts). Cardiovasc Pathol 1992;1:211-217.

17. Yeh T Jr, Ramaciotti C, Leonard SR, Roy L, Nikaidoh H. The aortic translocation (Nikaidoh) procedure: midterm results superior to the Rastelli procedure. J Thorac Cardiovasc Surg 2007;133:461-469.

18. Coselli JS, LeMaire SA, Preventza O, de la Cruz KI, Cooley DA, Price $\mathrm{MD}$, et al. Outcomes of 3309 thoracoabdominal aortic aneurysm repairs. J Thorac Cardiovasc Surg 2016;151:1323-1337.

19. Park SJ, Kim JB. An eight-branched aortic graft for reconstruction of visceral and intercostal arteries during extent II thoraco-abdominal aortic surgery. Eur J Cardiothorac Surg 2018;53:1282-1283.

20. David TE, Feindel CM. An aortic valve-sparing operation for patients with aortic incompetence and aneurysm of the ascending aorta. J Thorac Cardiovasc Surg 1992;103:617-621.

21. Erasmi AW, Sievers HH, Bechtel JF, Hanke T, Stierle U, Misfeld M. Remodeling or reimplantation for valve-sparing aortic root surgery? Ann Thorac Surg 2007;83:S752-S756

22. Maselli D, Weltert L, Scaffa R, Nardella S, Guerrieri Wolf L, De Paulis R. Differences in aortic cusp coaptation between the reimplantation and the remodeling techniques of aortic valve-sparing surgery: an in vitro porcine model study. J Thorac Cardiovasc Surg 2014;147:615-618.

23. Kim DH, Handschumacher MD, Levine RA, Sun BJ, Jang JY, Yang DH, et al. Aortic valve adaptation to aortic root dilatation: insights into the mechanism of functional aortic regurgitation from 3-dimensional cardiac computed tomography. Circ Cardiovasc Imaging 2014;7:828-835.

24. Yang DH, Kim DH, Handschumacher MD, Levine RA, Kim JB, Sun BJ, et al. In vivo assessment of aortic root geometry in normal controls using 
3D analysis of computed tomography. Eur Heart J Cardiovasc Imaging 2017;18:780-786.

25. Hermsen JL, Burke TM, Seslar SP, Owens DS, Ripley BA, Mokadam NA, et al. Scan, plan, print, practice, perform: development and use of a patient-specific 3-dimensional printed model in adult cardiac surgery. J
Thorac Cardiovasc Surg 2017;153:132-140.

26. Shiraishi I, Yamagishi M, Hamaoka K, Fukuzawa M, Yagihara T. Simulative operation on congenital heart disease using rubber-like urethane stereolithographic biomodels based on 3D datasets of multislice computed tomography. Eur J Cardiothorac Surg 2010;37:302-306. 